\title{
Comparison of 3-day and 7-day recall periods for food consumption reference values in foodborne disease outbreak investigations
}

\section{Original Paper}

Cite this article: Morton VK, Thomas MK, Ciampa N, Cutler J, Hurst M, Currie A (2019). Comparison of 3-day and 7-day recall periods for food consumption reference values in foodborne disease outbreak investigations. Epidemiology and Infection 147, e129, 1-4. https://doi.org/10.1017/S0950268818003370

Received: 3 April 2018

Revised: 29 September 2018

Accepted: 7 November 2018

Key words:

Foodborne infections; outbreaks

Author for correspondence: V. K. Morton, E-mail: Vanessa.morton@canada.ca (c) The Author(s) 2019. This is an Open Access article, distributed under the terms of the Creative Commons AttributionNonCommercial-NoDerivatives licence (http:// creativecommons.org/licenses/by-nc-nd/4.0/), which permits non-commercial re-use, distribution, and reproduction in any medium, provided the original work is unaltered and is properly cited. The written permission of Cambridge University Press must be obtained for commercial re-use or in order to create a derivative work.

\section{CAMBRIDGE} UNIVERSITY PRESS
V. K. Morton ${ }^{1}$, M. K. Thomas ${ }^{1}$, N. Ciampa ${ }^{1}$, J. Cutler ${ }^{1}$, M. Hurst ${ }^{1}$ and A. Currie ${ }^{2}$

${ }^{1}$ Public Health Agency of Canada, Guelph, ON, Canada and ${ }^{2}$ Public Health Agency of Canada, Ottawa, ON, Canada

\begin{abstract}
Investigations into an outbreak of foodborne disease attempt to identify the source of illness as quickly as possible. Population-based reference values for food consumption can assist in investigation by providing comparison data for hypothesis generation and also strengthening the evidence associated with a food product through hypothesis testing. In 2014-2015 a national phone survey was conducted in Canada to collect data on food consumption patterns using a 3- or 7-day recall period. The resulting food consumption values over the two recall periods were compared. The majority of food products did not show a significant difference in the consumption over 3 days and 7 days. However, comparison of reference values from the 3day recall period to data from an investigation into a Salmonella Infantis outbreak was shown to support the conclusion that chicken was the source of the outbreak whereas the reference values from a 7-day recall did not support this finding. Reference values from multiple recall periods can assist in the hypothesis generation and hypothesis testing phase of foodborne outbreak investigations.
\end{abstract}

\section{Introduction}

Population-based reference values for food consumption can assist in foodborne disease outbreak investigations. These reference values can be employed to quickly compare food consumption among cases to the general population, providing evidence to identify a specific food item or items to be investigated further. Traditionally, analytical epidemiological studies such as cohort or case-control studies have been employed to identify the source in foodborne disease outbreak investigations. These studies can be challenging to complete in an outbreak situation as they are time and resource intensive and may require specific technical knowledge. Population-based reference values can be an alternative to these studies; they can be used for hypothesis generation or strengthening the association of a food item with illness $[1,2]$.

Obtaining appropriate food exposure reference values can be challenging due to the time and cost involved in surveying a population, thus having values on hand for a variety of food items would be beneficial both for foodborne outbreak investigation and other studies. In Canada, national reference values for food exposures relevant to enteric disease outbreak investigation, based on a 7-day recall period, have recently been published in Foodbook - a national, population-based telephone survey conducted in 2014-2015 that assessed Canadians' food, water and animal exposures [3]. A 7-day period was chosen as it was typically use for hypothesis generating interviews of cases of foodborne disease during national outbreak investigations and was in line with other food consumption studies [4].

In foodborne outbreak investigations, the recall period used for food consumption history should reflect the incubation period of the pathogen (e.g. Escherichia coli has a 3-4 day median incubation period compared with Salmonella that has a 6-72 $\mathrm{h}$ incubation period) [5]. Ideally, the recall period used in case questionnaires should also be used for population-based reference values, to ensure appropriate comparisons.

The potential impact of different recall periods used among cases and population-based reference values in an outbreak investigation has not been thoroughly examined. Some research does exist however in comparing the impact of different recall periods when assessing risk factors for enteric illness infection. In Denmark, a prospective case-control study conducted from 1997 to 1999 that assessed risk factors for sporadic infection with Salmonella Enteritidis, found that the shorter the exposure period, the stronger the strength of association was for relevant risk factors [6]. For example, for eggs, a commonly consumed item in Denmark, the odds ratio was 2.2 when the day before onset of illness was used, compared with 1.6 for 3-days and 0.8 for 7-days. The authors concluded that stronger associations may be obtained by using exposure periods based on the most likely and common incubation period rather than the maximum incubation period. In a study conducted in Canada, where multiple exposures of healthy people to potential enteric pathogen sources were assessed by various criteria including differing 
recall periods, it was found that for a few exposures, the occurrence significantly increased with the length of the recall period [7]. This finding is also consistent with a study conducted across Europe, examining the influence of food consumption survey duration on estimates, where it was also found that consumption of particular food items increased with increasing time (i.e. ranging from 1 to 14 day recordings of food intake) and that the magnitude of the increase depended on how rarely or commonly a food was consumed [8].

As part of the Foodbook study, a sub-sample of respondents were asked about their food consumption exposures in the 3 days before interview instead of the past 7 days to explore the impact of a different recall period. This paper will use Foodbook survey data to examine if there is a statistically significant difference in the proportion of Canadians reporting exposure to specific food items over a 3-day vs. a 7-day recall period. As a secondary objective, it will also look at a historical Salmonella outbreak and apply the 3-day recall period consumption information to determine if there is a difference in exposures identified. This will inform the interpretation of comparisons of outbreak case data based on a 3-day recall period to Foodbook data based on a 7-day recall period during foodborne illness outbreaks.

\section{Methods}

Data were obtained from Foodbook, a national, population-based telephone survey. The survey was completed in all Canadian provinces and territories, from April 2014 to April 2015. The design and sampling methodology for the Foodbook study has been detailed elsewhere [3]. The majority of participants were asked about food exposures in the 7 days before interview. As a substudy, a small number of participants were selected to complete the survey using a 3-day recall period instead of a 7-day period. Participants were selected from the large study frame for this substudy to provide representation throughout the year and across provinces. Enrolment of participants was distributed across the week to limit the possible effect of day of the week on food consumption patterns.

Survey results were weighted to reflect the Canadian population. Survey data using a 7-day recall period were weighted based on geographic area, age group, gender, household type, number of individuals in the household and number of land lines and cell phones in the household [3]. Due to the small sample size for the 3-day recall sub-study, a simplified weighting method was used, based on age group and province/territory only.

Weighted proportions for each recall period were calculated using STATA, version 15.1 (StataCorp, College Station, Texas). Responses were combined to generate seven derived food categories (e.g. consumption of 'any ground beef was derived from responses to multiple ground beef consumption questions). Differences between the recall periods were calculated using the two-tailed adjusted Wald test; with a $P$-value threshold of 0.05 used to identify significant results. A Bonferroni $P$-value adjustment was applied to account for multiple testing.

\section{Outbreak example}

To assess the effect of 3-day vs. 7-day food recall on actual outbreak data, case food exposure information was obtained from an outbreak investigation of Salmonella Infantis. In 2015-2016, an outbreak of 110 confirmed cases of Salmonella Infantis in Canada was investigated by the Public Health Agency of
Canada, in conjunction with federal and provincial partners [9]. During the outbreak investigation, exposure information was analysed for 36 cases who were interviewed with an enhanced questionnaire regarding their food exposure in the 3 days prior to illness onset. The proportion of cases reporting each exposure (yes or probably) was compared with the Foodbook study's 3 -day reference values using an exact probability calculation. Significance was determined using a $P$-value threshold of $<0.05$.

\section{Results}

A total of 11139 surveys were completed during the Foodbook study; 10942 individuals were asked a 7-day recall period for food exposures and an additional 197 individuals were asked about a 3-day recall period. Each group was asked about exposure to food items (Supplementary Appendix 1). Participants were enrolled from all 13 provinces and territories in Canada. Participants were sampled across four age categories $(0-9,10$ $19,20-64,65+$ years) and province/territory so that a minimum sample would be available in each age group for each province/ territory. More females completed the 3-day recall period survey than males; 115 (58\%) vs. 82 (42\%) (Table 1). Surveys with the 3 -day recall were distributed throughout the year, and were completed on all seven days of the week.

The Foodbook survey asked about consumption of 193 food items. Of these, $15(8 \%)$ were consumed by $60 \%$ or more of the study population during a 3-day recall period; these were considered to be commonly consumed in the population. An additional 121 food items were considered to be rarely or uncommonly consumed in the Canadian population based on a 3-day recall period; $74(38 \%)$ items were consumed by $<10 \%$ of the population and an additional $47(24 \%)$ were consumed by $10-20 \%$ of the study population. Comparing the proportion of the population consuming these 193 food items over 3-day and 7-day recall periods, $30(16 \%)$ of these comparisons were significantly different (Supplementary Appendix 1). Among the common food items, $1(7 \%)$ was significantly different; for the uncommon and rare food items, $8(17 \%)$ and $16(22 \%)$ were significantly different, respectively.

\section{Outbreak example}

An analysis of case exposure data collected during a Salmonella Infantis outbreak and Foodbook reference showed considerable differences based on the recall period used (Table 2). When calculating significance of food items consumed by cases, only 'Chips or pretzels' was significantly higher among cases compared with population reference values using the 7 -day recall period. However, once the case exposure proportions were compared with the 3-day recall period reference values, five food items were significantly higher among the cases, including chicken (not including deli meat), which was ultimately identified as the source of the outbreak [9].

\section{Discussion}

Foodbook data provide a useful reference tool for enteric disease outbreak investigations by providing detailed reference values for food exposures based on a 7 -day recall period. Results from this sub-study of food exposures based on a 3-day recall period could also be used to inform outbreak investigations when case information is based on a 3-day recall period. 
Table 1. Description of survey participants asked about a 3-day and 7-day recall period by age and gender $(N=197)$

\begin{tabular}{|c|c|c|c|c|c|c|}
\hline \multirow[b]{2}{*}{ Age group (years) } & \multicolumn{3}{|c|}{ 3-day recall } & \multicolumn{3}{|c|}{ 7-day recall } \\
\hline & Male & Female & Total & Male & Female & Total \\
\hline $10-19$ & 11 & 7 & 18 & 1254 & 1113 & 2367 \\
\hline $20-64$ & 42 & 57 & 99 & 1202 & 1923 & 3125 \\
\hline Total & 82 & 115 & 197 & 4828 & 6104 & 10932 \\
\hline
\end{tabular}

Table 2. Abbreviated list of food exposures reported by cases in a national outbreak of Salmonella Infantis in 2015 compared with Foodbook reference values for 3-day and 7-day recall periods (2014-2015)

\begin{tabular}{|c|c|c|c|c|c|}
\hline Food item & $\begin{array}{c}\text { Cases exposed } \\
\text { (3-day exposure period) } \\
N(\%)\end{array}$ & $\begin{array}{l}\text { 3-day exposure } \\
\text { period (\%) }\end{array}$ & $\begin{array}{l}\text { 7-day exposure } \\
\text { period }(\%)\end{array}$ & $\begin{array}{c}P \text {-value, } \\
\text { 3-day }\end{array}$ & $\begin{array}{c}P \text {-value, } \\
\text { 7-day }\end{array}$ \\
\hline Any carrots (including baby and mini) & $18(69)$ & 75 & 81 & 0.812 & 0.960 \\
\hline Apples & $19(68)$ & 60 & 72 & 0.253 & 0.772 \\
\hline Cheddar cheese & $23(77)$ & 59 & 73 & 0.036 & 0.394 \\
\hline Chips or pretzels & $22(73)$ & 43 & 54 & 0.001 & 0.023 \\
\hline Onions & $19(66)$ & 72 & 83 & 0.843 & 0.993 \\
\hline Tomatoes & $20(69)$ & 66 & 73 & 0.474 & 0.760 \\
\hline Pork (not including deli-meats) & $22(71)$ & 34 & 55 & $<0.001$ & 0.053 \\
\hline Lettuce or leafy greens & $20(69)$ & 81 & 82 & 0.959 & 0.978 \\
\hline Eggs & $24(77)$ & 68 & 79 & 0.170 & 0.696 \\
\hline
\end{tabular}

Bold indicates these results are significant at the critical $p$-value of 0.05 .

${ }^{a}$ Source of illness in this outbreak was ultimately identified as chicken.

Reference food exposure data based on a 3-day recall period can be useful for outbreaks associated with commonly consumed food items. Although very few common food items are significantly different when comparing 3-day to 7-day, there are differences between the food consumption reported in these groups, this difference may be significant when compared with exposure frequencies reported by outbreak cases. This could be important for outbreak investigations as a high frequency of a typically 'rare' food item would naturally stimulate further investigation, while a food item thought to be more common may not. Using the 3-day recall estimate for some pathogens, focuses the comparison on the most relevant time period for infection and provides a more accurate comparator to case exposure information collected with the same recall period.

The outbreak example demonstrates the utility of food exposure estimates based on a 3-day recall period in practice. Outbreak cases were asked about exposures using a 3-day recall period and so comparing these values with the 3-day reference value was most appropriate. In this situation the implicated food was a common exposure, chicken, a difference in the statistical associations was observed when using the 3-day and 7-day recall period reference values.
Reference values for food exposures based on both 3-day and 7-day recall periods provide important data to help investigators identify the cause of, and take action in, foodborne illness outbreaks. While analytical studies are the gold standard epidemiologic approach to identify causes of infection, they are expensive, time-consuming and often not practical nor required in the context of a fast-paced, community wide outbreak investigation. A primary aim of foodborne illness investigators is to gather and weigh the evidence for suspect foods as the source of infections so that control measures can be implemented $[10,11]$. Comparing population food exposure data with those gathered from outbreak cases provides vital clues that can be used to generate hypotheses regarding the source of an outbreak. In addition, these comparisons provide evidence that when combined with other information from the epidemiological, laboratory and food safety investigations can be used to draw strong conclusions regarding the vehicle of infection. Food exposure data based on a 3-day recall window will increase investigators ability to interpret and draw conclusions based on the totality of evidence in a foodborne illness outbreak investigation, particularly when the incubation period is short and the suspect food exposure is a commonly consumed food item. 
Several limitations exist, with the most important being the small sample size $(n=197)$ for the 3 -day recall responses. This limits our ability to examine trends in food exposure by demographics or province/territory. Also, since survey respondents were only asked about a 3-day recall period or a 7-day recall period, future work could involve asking about consumption over multiple time periods $(1,3,7,14$ days, etc.), to facilitate additional analyses and comparisons of recall periods.

Using the same recall period for exposures of cases and reference values is important for identifying the most important associations. As different pathogens have different incubation periods, there are times when a 7-day recall period may not be the best choice and a 3-day recall period may be more appropriate. Exposure values based on a 3-day recall period can be used by outbreak investigators who have case exposure data based on the same time period. Results of this analysis using case exposure data and population reference values with the same recall period showed that commonly consumed foods often had lower food consumption proportions for the 3-day recall period vs. the 7-day values, which has an impact on identifying suspect food exposure. Further research based on a larger sample size and having respondents asked of multiple recall periods could provide additional evidence related to this topic.

Supplementary material. The supplementary material for this article can be found at https://doi.org/10.1017/S0950268818003370.

Author ORCID. (D) Vanessa Morton, 0000-0002-4471-1985

Acknowledgements. The authors thank the Centre for Foodborne, Environmental and Zoonotic Infectious Diseases (Public Health Agency of Canada), Outbreak Management Division and Food-Borne Disease and Antimicrobial Resistance Surveillance Division, provincial and territorial epidemiologists for their assistance with the Foodbook project; the staff at R.A. Malatest \& Associates Ltd. for their expert interviewing; and the Foodbook respondents for their participation. Thanks are also to Tanis Kershaw, Christine Gardhouse and Florence Tangauy for assistance with the Salmonella Infantis outbreak data.

Financial support. This work was supported by the Public Health Agency of Canada.

Conflict of interest. None of the authors has conflict of interest.

\section{References}

1. Gaulin C et al. (2012) Escherichia coli O157:H7 outbreak linked to raw milk cheese in Quebec, Canada: use of exact probability calculation and casecase study approaches to foodborne outbreak investigation. Journal of Food Protection 75, 812-818.

2. Tataryn J et al. (2014) Outbreak of E. coli O157:H7 associated with lettuce served at fast food chains in the Maritimes and Ontario, Canada, Dec 2012. Canada Communicable Disease Report 40 (Suppl 1), 2-9.

3. Public Health Agency of Canada (2015) Foodbook Report. Available at https://www.canada.ca/en/public-health/services/publications/food-nutrition/foodbook-report.html.

4. Centers for Disease Control and Prevention (CDC) (2006-2007) Foodborne Active Surveillance Network (FoodNet) Population Survey Atlas of Exposures. Atlanta, Georgia: U.S. Department of Health and Human Services, Centers for Disease Control and Prevention. Available at https://www.cdc.gov/foodnet/surveys/foodnetexposureatlas0607_508. pdf

5. Heymann DL (ed.) (2014) Control of Communicable Diseases Manual, 20th Edn. Washignton: American Public Health Association.

6. Molbak K and Neimann J (2002) Risk factors for sporadic infection with Salmonella Enteritidis, Denmark, 1997-1999. American Journal of Epidemiology 156, 654-661.

7. David JM et al. (2014) Assessing multiple foodborne, waterborne and environmental exposures of healthy people to potential enteric pathogen sources: effect of age, gender, season, and recall period. Epidemiology and Infection 142, 28-39.

8. Lambe J et al. (2000) Enhancing the capacity of food consumption surveys of short duration to estimate long-term consumer-only intakes by combination with a qualitative food frequency questionnaire. Food Additives and Contaminants 17, 177-187.

9. Public Health Agency of Canada (2016) Multi-provincial outbreak of Salmonella Infantis: Final Epidemiological Summary. Government of Canada.

10. Health Canada, Public Health Agency of Canada, Canadian Food Inspection Agency (2011) Weight of evidence: factors to consider for appropriate and timely action in a foodborne illness outbreak investigation. Ottawa: Health Canada. https://www.canada.ca/content/dam/hcsc/migration/hc-sc/fn-an/alt_formats/pdf/pubs/securit/2011-food-illnessoutbreak-eclosion-malad-ailments-eng.pdf.

11. Council to Improve Foodborne Outbreak Response (CIFOR) (2009) Guidelines for Foodborne Disease Outbreak Response. Atlanta: Council of State and Territorial Epidemiologist; 2009. https://cifor.us/products/ guidelines 